\title{
Restraining and encouraging the use of hand gestures: Effects on speech
}

\author{
Alice Cravotta ${ }^{1}$, Maria Grazia Busà ${ }^{1}$, Pilar Prieto ${ }^{2,3}$ \\ ${ }^{1}$ Università degli Studi di Padova, Italy; Dipartimento di Studi Linguistici e Letterari (DiSLL) \\ ${ }^{2}$ Universitat Pompeu Fabra, Barcelona, Catalunya; Departament de Traducció i Ciències del \\ Llenguatge \\ ${ }^{3}$ Institució Catalana de Recerca i Estudis Avançats, ICREA, Barcelona, Catalunya \\ alice.cravotta@phd.unipd.it, mariagrazia.busa@unipd.it, pilar.prieto@upf.edu
}

\begin{abstract}
Previous studies have investigated the effects of the inability to make hand gestures on speakers' fluency; however, the question of whether encouraging speakers to gesture affects their fluency has received little attention. This study investigates the effect of restraining (Experiment 1) and encouraging (Experiment 2) hand gestures on the following correlates of speech: speech discourse length (number of words and discourse length in seconds), disfluencies (filled pauses, self-corrections, repetitions, insertions, interruptions, silent pauses), and acoustic properties (speech rate, measures of intensity and pitch). In two experiments, 10 native speakers of Italian took part in a narration task where they were asked to describe comic strips. Each experiment compared two conditions. In Experiment 1, subjects first received no instructions as to how to behave when narrating. Then they were told to sit on their hands while speaking. In Experiment 2, subjects first received no instructions and were then actively encouraged to use hand gestures. The results showed that restraining gestures leads to quieter and slower paced speech, while encouraging gestures triggers longer speech discourse, faster speech rate and more fluent and louder speech. Thus, both restraining and encouraging hand gestures seem to clearly affect prosodic properties of speech, particularly speech fluency.
\end{abstract}

Index Terms: gestures, fluency, encouraging gestures, restraining gestures, disfluencies, prosody.

\section{Introduction}

Research on the relationship between speech and co-speech gestures has shown that the two modalities form a single integrated system [1][2]. Gesture-speech interactions have been explained by a variety of models (e.g., [3][4][5][6][7][8][9]). Additionally, both behavioral and neuroimaging studies of speech and co-speech gesture production have suggested that spoken language and arm gestures are controlled by the same motor control system [10][11]. Moreover, there is general agreement on gestures' self-oriented cognitive functions [12] and their beneficial role in learning, thinking and speaking (e.g., learning [13][14], solving tasks [15], lightening cognitive and memory load [16][17] and creative thinking [18]). Thus, intuitively enough, both restraining and encouraging the use of gestures should have an impact on speakers' speech production. While previous studies have mostly focused on the effects that the inability to gesture has on speakers' fluency, the question of how encouraging speakers to gesture might affect their fluency has thus far attracted little attention.

Previous studies have shown that the inability to gesture impacts speech production in various ways. One very early study [19] (which now seems virtually anecdotal) found that speech becomes less fluent and more monotonous when the speaker is unable to gesture. A number of more recent studies have found speech under these conditions to be, in general, less fluent [20][21][22], especially in expressing spatial content [3], which becomes less detailed and less semantically rich [23]. On the other hand, Rimé et al. [24] did not find speech to be less fluent but did report a decrease in general imagery content. And Hoetjes et al. [25] could not confirm any clear effects either on speech fluency or on speech monotony.

As for encouraging gestures, to our knowledge no previous studies have directly addressed the question of whether asking participants to gesture while speaking has any impact on speech fluency. Nevertheless, previous findings suggest that the use of gestures enhances the voice spectrum of the semantically related word [26] and the acoustic realization of prosodic prominence [27]. Also, asking speakers to gesture has been found to have a positive effect on lexical retrieval [28] and, in the case of children, it enhances their creative thinking [18].

In the present work we investigate the effect of restraining (Experiment 1) and encouraging (Experiment 2) the use of gestures in a narration task. We will analyze the resulting narratives through various prosodic parameters: speech discourse length (number of words and discourse length in seconds); disfluency (number of filled pauses, self-corrections, repetitions, insertions, interruptions, silent pauses); and acoustic properties (speech rate and measures of intensity and pitch). The aim is to provide more detailed evidence of the effects of restraining gestures on various aspects of speech prosody, as well as to start exploring the issue of whether encouraging the use of gestures has an effect on speech prosody too.

\section{Experiment 1: Restraining gestures}

Experiment 1 aimed to test whether the inability to gesture changes prosodic aspects of speech (specifically discourse length, fluency, speech rate, pitch and intensity) in a narration task with two within-subject conditions: telling the story with no instructions regarding how to behave when narrating (no instruction condition, henceforth $\mathrm{N}$ condition), and telling the story while not being able to gesture (restrained gestures condition, henceforth $\mathrm{R}$ condition). 


\subsection{Methods}

\subsubsection{Participants}

Ten female native speakers of Italian participated in the experiment. They were all from the Veneto region (age $\mathrm{M}=$ 23.2; $\mathrm{SD}=1.2$ ) and most of them were undergraduate students at the University of Padua. As compensation for their participation they were either given course credits or a free breakfast.

\subsubsection{Materials}

Fourteen four-scene comic strips adapted from Simon's Cat by Simon Tofield were used to elicit speech (see Figure 1). The strips were carefully selected and adapted so that they were equivalent in terms of complexity and length. The 14 strips were divided into two sets of seven, each set to be used in one of two conditions. For both conditions, two of the strips were reserved for initial task familiarization purposes, while the remaining five were to serve as stimuli for the experiment proper.
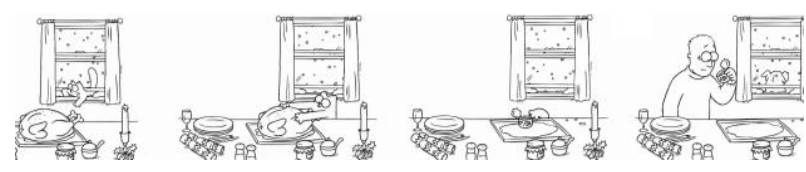

Figure 1: Example of a 4-scene comic strip used for the experiment (from Simon's Cat by Simon Tofield, reproduced with permission).

\subsubsection{Procedure}

Participants were tested individually in a quiet room at the University of Padua with the help of a research assistant (male, 29, also from the Veneto region). Each session was recorded with a HD video camera (JVC GZ-HD7E Everio) and speech was recorded as a separate audio track using a MIPRO wireless microphone with a bodypack transmitter connected to a Zoom R16 digital audio mixer. The participants were first given the following written instructions (translated from Italian): "You will be shown a set of short-sequence comic strips. A cat and its friends are the protagonists. Take your time to look at each of the short strips. When you think you understand the story they depict, the comic strip will be covered up. Then you will have to describe the story in sufficient detail that your partner (who does not know the story) will later be able to reconstruct it by placing the four images that make up the strip in the correct order". Then, since the experiment had a within-subject design, each subject performed all 14 trials, seven in a "No instructions" condition $(\mathrm{N})$ and seven in a "Restrained gestures" condition (R). Each trial consisted of a three-step sequence: (1) the subject studied a four-scene comic strip to learn the story it depicted (2) the strip was concealed and the subject recounted the story to the research assistant; (3) the assistant attempted to reconstruct the story as told by the subject by putting the four images from the original strip in the correct order. Thus, participants began with the $\mathrm{N}$ condition, which consisted of two practice trials and five target trials, which were recorded for subsequent data analysis. Then the participants repeated the procedure (using different comic strips) for the $\mathrm{R}$ condition, in which they were instructed to sit on their hands (as in [25]) while telling each story to prevent them from making hand gestures (see Figure 2, left-hand photos). Again, the last five trials were recorded for data. Participants were also made to believe that the research assistant was a fellow participant and did not know the stories in advance. This was accomplished by giving him written instructions at the same time the subject was given hers. The intention here was to avoid effects of common ground and to give face validity to the narration task: the subjects felt an obligation to explain the story clearly and fully because their "fellow participant" was dependent on them to understand it.
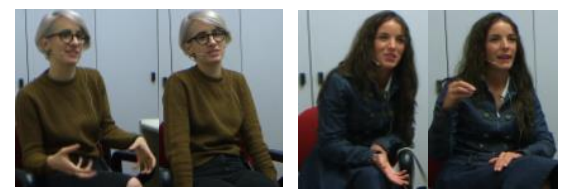

Figure 2: Picture stills of participants telling stories in the control and experimental conditions. From left: Experiment $1(N$ and $R)$ and Experiment $2(N$ and $E)$.

Audio recordings of a total of 100 short narrations were obtained (10 participants $\times 10$ target trials) lasting a total of 36.02 minutes (18.14 minutes in the $\mathrm{N}$ condition and 17.87 in the $\mathrm{R}$ condition).

\subsubsection{Data analyses \\ 2.1.4.1 Speech discourse length}

The recordings were cut so that a separate short audio file was created for each story told. After the duration in seconds of each file was measured, the contents were manually transcribed and the number of words per story counted.

\subsubsection{Fluency and disfluency measures}

Fillmore et al. [30, p. 93] define fluency as "the ability to talk at length with few pauses, (...) to fill time with talk. A person who is fluent (...) does not have to stop many times to think of what to say next or how to phrase it". In addition, according to Zellner [29, p. 48] "people are disfluent if they often hesitate, make non-functional pauses and make speech errors and selfcorrections." Thus, fluency is usually measured not only by speech rate but also by the absence of what characterizes disfluency. For this reason, based on previous studies [31][32][33], instances of any of the following disfluencies were recorded as inversely indexing fluency.

- Filled pauses (sounds like “ehm”, “mmm”);

- Self-corrections: syntax-based (e.g., rephrasing); lexicon-based (a word is replaced with another word); phonology-based (slip of the tongue/unclear pronunciations);

- Repetitions: of sounds (e.g., stuttering), words or longer segments;

- Insertions: of words or phrases when speech needs further qualification or detail;

- Interruptions: abrupt interruption of a word, or pronunciation of an isolated incoherent sound;

- Silent pauses: annotated automatically by a Praat script described in [34].

The absolute count of all types of disfluencies was converted into a relative measure (e.g., number of filled pauses per 100 words). 


\subsubsection{Acoustic properties}

Acoustic analysis of speech was performed using the Praat software [35]. A measure of speech rate was obtained using the script described in [34]. The script detects potential syllable nuclei in terms of peaks in intensity $(\mathrm{dB})$ that are preceded and followed by dips in intensity. It then divides the number of syllables produced in each audio file by the file's total duration (i.e., speech rate is given as number of syllables over duration). A set of intensity and pitch measures (mean, minimum, maximum and standard deviation, the last as a measure of intensity and pitch variability) were also extracted with Praat for every audio file.

\subsubsection{Statistical analyses}

Twenty-two GLMMs [36] were run using SPSS Statistics 24.0 (SPSS Inc., Chicago IL). A model was run for each of the following response variables: (1) story duration (s), (2) total number of words, (3) filled pauses, (4) syntactical (5) lexical and (6) phonological self-corrections, (7) repetitions, (8) insertions, (9) interruptions, (10) total number of selfcorrections, (11) total number of disfluencies, (12) total number of disfluencies (including silent pauses), and (13) total number of disfluencies (filled and silent pauses excluded); (14) speech rate, (15) mean intensity, (16) minimum intensity, (17) maximum intensity, (18) intensity standard deviation, (19) mean pitch, (20) minimum pitch, (21) maximum pitch and (22) pitch standard deviation. The models, with Participant as random factor, were used to estimate the effects of Condition (two levels: No instructions and Restrained gestures) on the variables described above.

\subsection{Results}

Out of the 22 GLMMs performed, only two showed a statistically significant effect of Condition, namely speech rate $(\mathrm{F}(1,98)=16.7, \mathrm{p}<.01)$ and mean intensity $(\mathrm{F}(1,98)=9.93$ $\mathrm{p}<.01)$. As Figure 4 shows, this main effect indicates a significantly lower speech rate and mean intensity in the $\mathrm{R}$ condition than in the $\mathrm{N}$ condition. No other significant main effects were found.

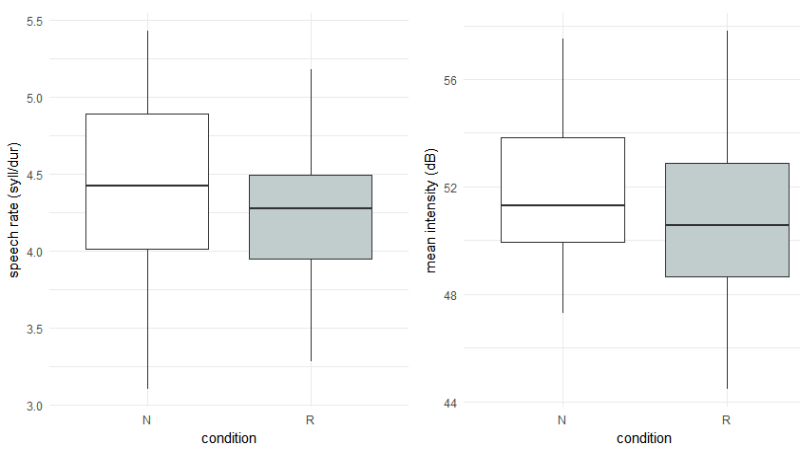

Figure 3: Box plots representing speech rate and mean intensity, the two variables that significantly differed, across the two conditions ( $N$ and $R$ ).

\section{Experiment 2: Encouraging gestures}

Experiment 2 aimed to test whether encouraging the use of gestures would have any impact on a set of prosodic aspects of speech (speech discourse length, fluency, speech rate, pitch and intensity) of Italian participants in a narration task.

\subsection{Methods}

The experiment followed exactly the same methodology as Experiment 1, the only difference being the subjects who participated and the experimental condition tested.

\subsubsection{Participants}

A different set of ten female native speakers of Italian participated in the experiment. They were also from the Veneto region (age $\mathrm{M}=26.2 ; \mathrm{SD}=3.1$ ), and again most of them were undergraduate students from the University of Padua. They were either given course credits or a free breakfast as compensation for their participation.

\subsubsection{Materials}

As in Experiment 1, a different set of 14 comic strips featuring Simon's Cat were selected to be used as stimuli to elicit speech (see section 2.1.2).

\subsubsection{Procedure}

Experiment 2 followed the same procedure described in section 2.1.3, with the same research assistant participating. Experiment 2 likewise had a within-subject design with two conditions. In this case, however, although the control condition (N) was identical to that used in Experiment 1 (no instructions given about how to retell the story), in the experimental condition participants were explicitly encouraged to use hand gestures while narrating (E condition). Their written instructions (translated from Italian) were: "Tell each story and use hand gestures to help you do so" (see Figure 2, right-hand photos). Participants were reminded to do so when they seemed to forget the prompt of using their hands in E condition. As in Experiment 1, audio recordings of a total of 100 short narrations were obtained (10 participants $\times 10$ target trials) lasting a total of 36.79 minutes (17.59 minutes in the $\mathrm{N}$ condition and 19.19 in the E condition).

\subsubsection{Data and statistical analyses}

Data analyses and statistical analyses for Experiment 2 were performed exactly as in Experiment 1 (see section 2.1.4).

\subsection{Results}

Out of the 22 GLMM models performed, the following 11 variables showed a statistically significant effect of Condition. First, a significant effect was found for story duration $(\mathrm{F}(1,98)$ $=10, \mathrm{p}<.01)$ with longer speech produced per story in the $\mathrm{E}$ than in the $\mathrm{N}$ condition. Word count per story was also found to be significantly higher in the $\mathrm{E}$ than in the $\mathrm{N}$ condition ( $\mathrm{F}$ (1, $98)=14.54, p<.01)$. As for the measures of fluency, significant effects were found for the number of filled pauses $(\mathrm{F}(1,90)=$ $10.93, p<.01)$, insertions $(\mathrm{F}(1,26)=12.91, \mathrm{p}<.01)$ and selfcorrections $(\mathrm{F}(1,52)=7.97, \mathrm{p}<.01)$, with lower occurrence in the $\mathrm{E}$ condition than in the $\mathrm{N}$ condition. It is important to note that filled pauses, insertions and self-corrections represent $90.2 \%$ of the total number of disfluencies annotated (or $49.4 \%$ of disfluencies if silent pauses $(45.2 \%)$ are included). A significant effect was also found for speech rate $(\mathrm{F}(1,98)=4.1$ $\mathrm{p}<.05)$, which was higher in the E condition. All the measures of intensity turned out to be significantly different across the 

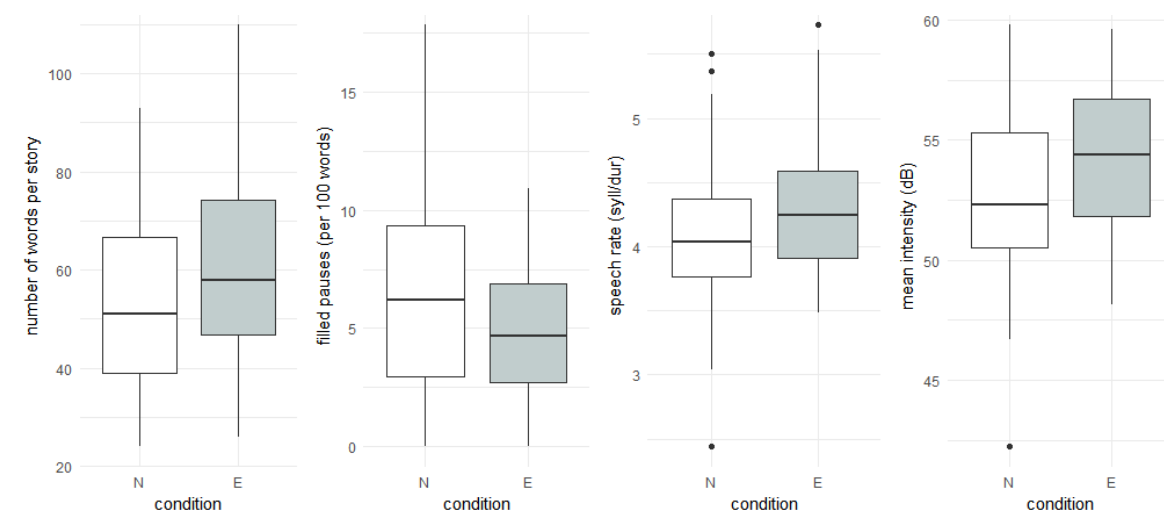

Figure 4 Box plots representing the main variables that significantly differed across the two conditions: No instructions $(N)$ and Encouraging gestures $(E)$.

two conditions: mean intensity $(\mathrm{F}(1,98)=22.37, \mathrm{p}<.01)$, minimum intensity $(\mathrm{F}(1,98)=7.8, \mathrm{p}<.01)$, maximum intensity $(\mathrm{F}(1,98)=15.19, \mathrm{p}<.01)$ and intensity variability $(\mathrm{F}(1,98)=$ $10.83, \mathrm{p}<.01)$. These results show that speech was louder in the $\mathrm{E}$ condition, while only intensity variability was higher in the $\mathrm{N}$ condition. No significant effects were found for any measure of pitch. To summarize, encouraging the use of gestures seems to have triggered longer speech discourse, a faster speech rate and more fluent and louder speech.

\section{Discussion}

The present study investigated the effects of restraining and encouraging the use of hand gestures on speech prosody. We observed that both conditions tend to affect various aspects of speech production.

In Experiment 1 we found that preventing participants from making hand gestures affected both speech rate and loudness. In particular, speakers not allowed to gesture tended to speak at a slower pace and less loudly. These findings are in line with previous studies claiming that the inability to gesture has a negative effect on speech fluency, of which one of the main components is speech rate. However, in our findings restricting gestures did not significantly affect the number of disfluencies produced. This might be due to the relatively small number of participants and the exploratory nature of this study. Given previous findings suggesting that an inability to gesture inhibits speech fluency only when the discourse includes spatial content phrases [3], a more detailed analysis of the relation between spatial content and the number of disfluencies might clarify our results. In addition, our results suggest that restraining gestures reduces speech loudness. In previous investigations very little attention has been paid to the potential effects of gesture inability on voice intensity, and we believe that this aspect merits further investigation.

In Experiment 2, we observed that encouraging speakers to move their hands had a number of effects on the prosodic aspects of speech, namely it lead to (a) longer discourses, (b) faster pace, (c) fewer disfluencies and (d) greater volume. In other words, the prompt to use hand gestures seems to have enhanced speech fluency and other prosodic features related to expressiveness. It is worth noting here that the extent to which participants moved their hands simply because they were prompted to do so has yet to be verified quantitatively. A visual inspection of the recorded data suggests that the prompts worked quite well, consistent with some previous studies
[37][38]. However, Parrill et al. [39], for instance, noted that such prompting did not always lead to a higher gesture rate. Be that as it may, when encouraged to move their hands, speakers spent more time and used more words to tell each story, indicating that asking participants to gesture somehow influenced how they approached the narrative act. The number of words used and duration of speech might be related to the wider notion of speech fluency proposed by Fillmore et al. [30, p. 93]: "Fluency is the ability to talk in coherent, reasoned and 'semantically dense' sentences" and "to be creative and imaginative in (...) language use". An interesting follow-up of the study would be to further analyze how encouraging the use of gesture affects lexicon and syntax: Does encouraging the use of hand gestures enhance the semantic density of speech? Does it lead to more creative and imaginative speech? Experiment 2 also showed that encouraging participants to gesture somehow enhanced their speech in that it became more fluent and louder. These findings need to be further investigated: can they be better explained as a consequence of cognitive processes or are they more related to motor control? Stronger evidence in this direction would be coherent with the claim that speech and gesture involve shared motor control systems.

In general, though our findings come from two experiments based on a rather small sample, they are nonetheless consistent with previous findings and theoretical models of speech and gesture production; if gestures have a role in speech production, it should not be surprising that either restraining or encouraging gestures will affect the prosodic properties of speech to some extent. The results reported here suggest that not only restraining hand gesture, but also encouraging participants to move their hands while speaking can show new and interesting interactions between gestures and prosody that need to be investigated.

\section{Acknowledgements}

This collaborative research was the result of an ErasmusPlus Inter-Institutional Mobility Agreement between the University of Padua and Universitat Pompeu Fabra. We are grateful to Nicolò Marcato for help with recordings and also serving as the "fellow participant" during the experiment. We thank Joan Borràs-Comes for assistance with the statistical analysis, and Judith Llanes-Coromina for her work during the initial stages of the project. We also want to thank the Simon's Cat team for graciously allowing us use of their comic strips. Authors 
acknowledge funding from the University of Padua's Doctoral School of Linguistic, Philological and Literary Sciences, the Spanish Ministry of Science and Innovation (grant FFI201566533-P), and a grant awarded by the Generalitat de Catalunya (2014SGR-925) to the Prosodic Studies Group.

\section{References}

[1] A. Kendon, "Gesticulation and speech: Two aspects of the process of utterance," The relationship of verbal and nonverbal communication, vol. 25, no. 1980, pp. 207-227, 1980.

[2] D. McNeill, Gesture and thought. University of Chicago press, 2008.

[3] F. H. Rauscher, R. M. Krauss, and Y. Chen, "Gesture, speech, and lexical access: The role of lexical movements in speech production," Psychological Science, vol. 7, no. 4, pp. 226-231, 1996.

[4] U. Hadar and B. Butterworth, "Iconic gestures, imagery, and word retrieval in speech," Semiotica, vol. 115, no. 1-2, pp. 147-172, 1997 .

[5] R. M. Krauss, "Why do we gesture when we speak?," Current Directions in Psychological Science, vol. 7, no. 2, pp. 54-60, 1998.

[6] R. M. Krauss, Y. Chen, and R. F. Gottesman, "Lexical gestures and lexical access: a process model," Language and gesture, vol. 2, p. 261, 2000.

[7] S. Kita, "How representational gestures help speaking," Language and gesture, vol. 1, 2000.

[8] M. W. Alibali, S. Kita, and A. J. Young, "Gesture and the process of speech production: We think, therefore we gesture," Language and cognitive processes, vol. 15, no. 6, pp. 593-613, 2000.

[9] J. P. De Ruiter, "Postcards from the mind: The relationship between speech, imagistic gesture, and thought," Gesture, vol 7, no. 1, pp. 21-38, 2007

[10] M. Gentilucci and R. D. Volta, "Spoken language and arm gestures are controlled by the same motor control system," The Quarterly Journal of Experimental Psychology, vol. 61, no. 6, pp. 944-957, 2008.

[11] L. Marstaller and H. Burianová, "A common functional neural network for overt production of speech and gesture," Neuroscience, vol. 284, pp. 29-41, 2015.

[12] S. Kita, M. W. Alibali, and M. Chu, "How do gestures influence thinking and speaking? The gesture-for-conceptualization hypothesis" Psychological Review, vol. 124, no. 3, pp. 245-66 2017.

[13] S. Goldin-Meadow and S. M. Wagner, "How our hands help us learn," Trends in cognitive sciences, vol. 9, no. 5, pp. 234-241, 2005.

[14] S. W. Cook, Z. Mitchell, and S. Goldin-Meadow, "Gesturing makes learning last" Cognition, vol. 106, no. 2, pp. 1047-1058, 2008.

[15] M. W. Alibali and S. Kita, "Gesture highlights perceptually present information for speakers," Gesture, vol. 10, no. 1, pp $3-28,2010$.

[16] S. Goldin-Meadow, H. Nusbaum, S. D. Kelly, and S. Wagner, "Explaining math: gesturing lightens the load.," Psychological Science, vol. 12, no. 6, pp. 516-22, 2001.

[17] S. W. Cook, T. K. Yip, and S. Goldin-, "Gestures, but not meaningless movements, lighten working memory load when explaining math," Language and cognitive processes, vol. 27, no. 4, pp. 594-610, 2012.

[18] E. Kirk and C. Lewis, "Gesture facilitates children's creative thinking," Psychological Science, vol. 28, no. 2, pp. 225-232, 2017.

[19] S. Dobrogaev, "Ucnenie o reflekse v problemakh iazykovedeniia [Observations on reflexes and issues in language study]," Iazykovedenie i materializm, pp. 105-173, 1929.
[20] E. Morsella and R. M. Krauss, "The role of gestures in spatial working memory and speech," The American Journal of Psychology, vol. 117, no. 3, pp. 411-24, 2004.

[21] S. Finlayson, V. Forrest, R. Lickley, and J. M. Beck, "Effects of the restriction of hand gestures on disfluency.," Proceedings of Diss, Gothenburg Papers in Theoretical Linguistics, vol. 90, pp. 21-24, 2003.

[22] J. A. Graham and S. Heywood, "The effects of elimination of hand gestures and of verbal codability on speech performance," European Journal of Social Psychology, vol. 5, no. 2, pp. 189$195,1975$.

[23] A. B. Hostetter, M. W. Alibali, and S. Kita, "Does sitting on your hands make you bite your tongue? The effects of gesture prohibition on speech during motor descriptions," in Proceedings of the Cognitive Science Society, 2007, vol. 29, no. 29.

[24] B. Rimé, L. Schiaratura, M. Hupet, and A. Ghysselinckx, "Effects of relative immobilization on the speaker's nonverbal behavior and on the dialogue imagery level," Motivation and Emotion, vol. 8, no. 4, pp. 311-325, 1984.

[25] M. Hoetjes, E. Krahmer, and M. Swerts, "Does our speech change when we cannot gesture?," Speech Communication, vol. 57, pp. 257-267, 2014.

[26] P. Bernardis and M. Gentilucci, "Speech and gesture share the same communication system.," Neuropsychologia, vol. 44, no. 2, pp. 178-90, 2006

[27] E. Krahmer and M. Swerts, "The effects of visual beats on prosodic prominence: Acoustic analyses, auditory perception and visual perception," Journal of Memory and Language, vol. 57, no. 3, pp. 396-414, 2007.

[28] C. Lucero, H. Zaharchuk, and D. Casasanto, "Beat gestures facilitate speech production," in Proceedings of the Cognitive Science Society, 2014, vol. 36, no. 36.

[29] B. Zellner, "Pauses and the temporal structure of speech," in Zellner, B.(1994). Pauses and the temporal structure of speech, in E. Keller (Ed.) Fundamentals of speech synthesis and speech recognition.(pp. 41-62). Chichester: John Wiley., John Wiley, 1994, pp. 41-62.

[30] C. J. Fillmore, D. Kempler, and W. S. Wang, Individual differences in language ability and language behavior. Academic Press, 2014.

[31] C. Bergmann, S. A. Sprenger, and M. S. Schmid, "The impact of language co-activation on L1 and L2 speech fluency," Acta psychologica, vol. 161, pp. 25-35, 2015.

[32] S. Götz, Fluency in native and nonnative English speech, vol. 53. John Benjamins Publishing, 2013.

[33] J. Kormos, Speech production and second language acquisition. Routledge, 2014.

[34] N. H. De Jong and T. Wempe, "Praat script to detect syllable nuclei and measure speech rate automatically," Behavior research methods, vol. 41, no. 2, pp. 385-390, 2009.

[35] P. Boersma, "Praat: doing phonetics by computer," http://www. praat. org/, 2006.

[36] B. T. West, K. B. Welch, and A. T. Galecki, Linear mixed models: a practical guide using statistical software. CRC Press, 2014.

[37] S. W. Cook, T. K. Yip, and S. Goldin-Meadow, "Gesturing makes memories that last," Journal of memory and language, vol. 63 , no. 4, pp. 465-475, 2010.

[38] M. Chu and S. Kita, "The nature of gestures' beneficial role in spatial problem solving," Journal of Experimental Psychology: General, vol. 140, no. 1, pp. 102-16, 2011.

[39] F. Parrill, J. Cabot, H. Kent, K. Chen, and A. Payneau, "Do people gesture more when instructed to?," Gesture, vol. 15, no. 3, pp. 357-371, 2016. 